\title{
Desempleo y sentimiento depresivo: un análisis motivacional y atributivo
}

\section{N. T. Feather y P. R. Davenport}

FUENTE: Journal of Personality and Social Psychology, 1981, 41, pp. 422-436.

\section{RESUMEN}

Se planteó la hipótesis, en base a la teoría de la expectativa-valencia, de que el sentimiento negativo subsiguiente al fracaso en conseguir un empleo sería mayor entre aquellos individuos que estaban fuertemente motivados a buscar empleo que entre los que lo estaban menos. Esta hipótesis se puso a prueba por medio de la administración de un cuestionario a una muestra de 212 jóvenes desempleados, contactados por medio de instituciones asistenciales de Adelaida (Australia del Sur). De acuerdo con la hipótesis, los resultados mostraron que los sujetos que indicaron en sus puntuaciones estar altamente motivados para conseguir un empleo, daban también puntuaciones más altas en sentimientos depresivos. Los sujetos con mayores niveles de sentimiento depresivo eran menos propensos a culparse a sí mismos por su desempleo y más propensos a culpar a las dificultades externas, tales como el estado de la situación económica. Asimismo dieron mayores puntuaciones de la valencia o atractivo percibido del trabajo. Sus puntuaciones retrospectivas referentes a la confianza que tenian en conseguir un trabajo cuando dejaron el colegio y sobre cuánto lo necesitaban y lo intentaron, tendian a ser también mayores que las de los sujetos menos deprimidos. Se discuten los resultados en relación con el enfoque de la expectativa-valencia, la teoría de la depresión de Beck y los recientes estudios sobre los vínculos afectivo-cognitivos, que emplean conceptos atributivos.

Han pasado más de cuarenta años desde que Eisenberg y Lazarsfeld (1938) publicaran su revisión de los efectos psicológicos del desempleo, revisión que resumía más de 100 estudios relacionados con el desempleo en la época de la Gran Depresión y en los años treinta. Describieron los efectos devastadores del desempleo en el bienestar psicológico para aquellos que lo sufrían: apatía, resignación, dudas sobre sí mismo, depresión, baja auto-estima, creencias fatalistas y cosas similares. Pusieron también de relieve la importancia de los estudios que 
relacionan los efectos psicológicos del desempleo con «una red de problemas teóricos interconectados» (p.385), de forma que la investigación pudiera guiarse por perspectivas teóricas en lugar de proceder de modo azaroso y no acumulativo. Jahoda (1979) nos ha recordado recientemente esta antigua advertencia en una discusión sobre el impacto del desempleo en los años 30 y 70 . Al igual que otros (p.e. Harrison, 1976; Hartley, 1980; Hyman, 1979; Gurney y Taylor, nota 1), comenta la ausencia relativa de investigación sistemática sobre el actual desempleo por parte de los psicólogos y pide que se hagan investigaciones teóricamente basadas sobre el daño psicológico que el desempleo puede causar y los medios de prevenir o aliviar este daño.

El presente estudio supone un paso en esta dirección, al investigar algunos de los correlatos del estado de ánimo depresivo entre los jóvenes desempleados. $\mathrm{Ya}$ que la investigación es de naturaleza correlacional, basada en la recogida de datos por medio de un cuestionario en un momento dado, no es posible llegar a conclusiones firmes sobre los antecedentes causales, pero esto no debe de considerarse como una deficiencia importante. Dada la poca información disponible sobre los efectos psicológicos del desempleo en los años 70 y 80 y la obvia importancia social del problema, la identificación de relaciones fiables entre variables es un primer paso necesario hacia un análisis causal más completo.

\section{Un enfoque de expectativa-valencia}

Los datos que hemos recogido son lo suficientemente ricos como para permitirnos probar algunas predicciones hechas a partir de un enfoque teórico general conocido como expectativa-valencia (Feather, en prensa). Esta teoría motivacional relaciona la acción con la atracción o aversión percibidas de los resultados esperados. Se considera que las acciones de una persona están relacionadas con las expectativas que la persona man- tiene y con los valores subjetivos (o valencias) de los resultados que pueden darse como consecuencia de la acción. El enfoque de la expectativa-valencia tiene una larga historia en la psicología. Las primeras contribuciones de Lewin, Totman, Rotter, Edwards y Atkinson fueron revisadas hace más de veinte años (Feather, 1959), y las más recientes discusiones sobre el enfoque general indican que continúan teniendo un status importante en las teorías de la acción (Feather, en prensa, Weiner, 1980). Los teóricos del aprendizaje social han puesto asimismo de relieve la importancia de tomar en consideración las consecuencias anticipadas en las explicaciones cognitivas de la conducta humana. Por ejemplo, las recientes aportaciones de Bandura (1977a, 1977b, 1978) sobre los principios del aprendizaje social, auto-regulación y auto-eficacia reconocen los efectos importantes que las consecuencias esperadas tienen sobre las acciones de la persona. En su análisis emplea una perspectiva teórica en la que se considera que el funcionamiento psicológico implica «una continua y reciproca interacción entre influencias conductuales, cognitivas y ambientales» (Bandura, 1978, p.345).

El enfoque de la expectativa-valencia implica que la fuerza de la motivación para buscar trabajo se relaciona tanto con las expectativas de que con esfuerzo se va a conseguir un empleo, como con el atractivo y aversión percibidas de la naturaleza del empleo en sí. Las expectativas pueden estar determinadas por una serie de factores diferentes, que incluyen de forma especialmente importante las percepciones que tiene una persona sobre su habilidad y destreza y sobre la facilidad o dificultad de la tarea. Por ejemplo, las personas que se ven a sí mismas con una competencia relativamente baja y que perciben que hay pocos trabajos asequibles, tenderán a tener expectativas de éxito bajas. Las valencias de los resultados están también relacionadas con un cierto número de variables, que incluyen la naturaleza de dichos resultados y sus posibles consecuencias (es decir, su ins- 
trumentalidad), los motivos dominantes de la persona y las fuerzas sociales y culturales que influencian la propia visión del trabajo. Una persona, por ejemplo, puede considerar atractivo un trabajo porque le aporta un salario alto, que puede ser usado de diversas maneras para satisfacer otros motivos (p.e., seguridad). Otra persona puede valorar el empleo porque la situación de trabajo le provee de oportunidades para la utilización de destrezas, afiliación, ejercicio del poder, etc., siendo todas cuestiones importantes en la vida de esa persona. Algunos trabajos se evitan porque tienen aspectos negativos (condiciones fisicas insatisfactorias, demasiada presión, etc.). Algunas culturas o subculturas valoran el trabajo más que otras, al concebirlo como una parte del ciclo vital que es esencial para el desarrollo de un sentimiento de identidad personal y de dignidad. Al mismo tiempo pueden considerar el trabajo como algo necesario para el avance de la sociedad como un todo. Podría esperarse también que estas diferencias culturales afecten al esquema de valencias.

Se supone que las expectativas y valencias, cualquiera que sea su origen, se combinan para determinar la fuerza de la tendencia para llevar a cabo un acto concreto, tal como buscar un tipo específico de empleo. Estas ideas no son nuevas. La teoria motivacional de la expectativavalencia ha sido aplicada a muchos campos, incluyendo áreas tan diversas como la conducta de logro, la adopción de decisiones, nivel de aspiración, teoria del aprendizaje social, trabajo y ejecución, conducta asistencial, conducta animal y conducta de búsqueda de información (ver Feather, en prensa, a, b y c para un tratamiento más amplio de estos puntos, y Vroom, 1964, para una aplicación pionera del análisis de expectativa-valencia a la situación del trabajo).

¿Cómo puede aplicarse este enfoque al análisis del sentimiento depresivo entre los parados? Se puede argumentar que el malestar consiguiente al fracaso en lograr un fin puede relacionarse con el atractivo global del fin que no se ha alcanzado (Feather, en prensa $a$, tabla 3.7.). El fracaso en conseguir un fin significa pérdida de los beneficios que el éxito pueda conferir, y el malestar incrementaría conforme mayor sea el atractivo neto del fin, es decir, mayor sea la pérdida ${ }^{1}$. Se puede asumir también que el malestar subsiguiente al fracaso será mayor cuando las expectativas de éxito sean inicialmente altas que cuando sean bajas. La no confirmación de una expectativa alta de éxito, al fracasar en la consecución de un fin, es probable que sea más aversiva que cuando la probabilidad subjetiva de éxito es muy baja (Feather, 1969 , en prensa $a$; Linsenmeier y Brickman, nota 2). Tomadas conjuntamente, estas suposiciones implican que el desagrado en torno al fracaso tenderá a ser mayor cuanto más motivada esté una persona para lograr un fin, dado el presupuesto común de que las expectativas y valencias se combinan multiplicativamente para determinar esta fuerza de la motivación. Así, el fracaso en alcanzar un fin estaría acompañado por un mayor grado de malestar cuando una persona tiene elevadas expectativas y percibe el objetivo como muy atractivo, que cuando una persona tiene bajas expectativas y percibe el objetivo como menos atractivo. En términos del enfoque de la expectativa-valencia, la primera persona debería estar más fuertemente motivada a perseguir el objetivo en cuestión. En aquellas condiciones en que las expectativas son altas pero el atractivo percibido es bajo, se podría esperar el encontrar niveles intermedios de malestar acerca del fracaso, correspondientes a niveles intermedios de motivación.

Por tanto, la predicción general que se deriva de este análisis es que el sentimiento negativo o depresivo subsiguiente al fracaso en obtener un empleo será mayor entre aquellos individuos que perciben el trabajo como atractivo, que tienen altas expectativas de lograr un empleo, y que están más fuertemente motivados a buscar un trabajo, en comparación con quienes tienen niveles más ba- 
jos de valencia, expectativa y motivación. Esta predicción se aplica a todos los estadios de la búsqueda de empleo, lo mismo iniciales que tardíos, aunque las expectativas de éxito y la motivación para buscar trabajo pueden ser relativamente bajas después de un fracaso continuado en encontrar empleo.

Los estudios anteriores sobre las reacciones afectivas al fracaso proveen de cierto apoyo a este análisis motivacional. Por ejemplo, Feather (1963) dejó que los sujetos trabajasen en un problema de "razonamiento perceptivo" insoluble, realizando tantos intentos como quisiesen antes de pasar a otro problema alternativo. Se encontró que las puntuaciones de malestar por el fracaso y de interés por el logro estaban positivamente correlacionadas $(\mathrm{r}=0,52, \mathrm{p}<0,001)$. Este resultado apoya la predicha relación positiva entre afecto negativo relacionado con el fracaso e intensidad de la motivación positiva. En el mismo estudio, las puntuaciones de malestar fueron mayores para los sujetos que al principio tenian más confianza que para aquellos que comenzaron la tarea con menores expectativas $(r=0,28, p<0,05)$. En un estudio posterior, que incluía la realización de anagramas, Feather (1969) encontró asimismo que los sujetos expresaban mayor insatisfacción cuando el fracaso en la superación de la prueba era inesperado que cuando se esperaba. Estos dos resultados apoyan la idea de que el sentimiento negativo es posible que sea mayor cuando las expectativas iniciales de éxito son altas que cuando son bajas, y la evidencia más reciente es también compatible con esta idea (Linsenmeier y Brickman, nota 2).

\section{Comparación con otras teorías}

Como podrá advertirse, el anterior análisis no trata del desarrollo de la depresión crónica o clínica, que puede generalizarse a muchos aspectos de la vida de la persona. El análisis se relaciona, más bien, con el sentimiento depresivo asociado con una condición específica, en concreto el fracaso en obtener un empleo, y el estudio interseccional que va a describirse está claramente planteado en este contexto. Es posible, sin embargo, que un paro prolongado pueda implicar en algunos casos una progresión desde el malestar y tristeza hacia formas más intensas y generales de depresión. Dado este hecho, sería absurdo ignorar las ideas de la teoría de la atribución y de la literatura clínica que tienen implicaciones para las formas 'más crónicas de depresión. Trataremos brevemente estas ideas, describiendo tres enfoques teóricos separados, pero relacionados entre si, volviendo después al tema de los efectos psicológicos de un prolongado desempleo.

Beck $(1967,1976)$, ha presentado una teoría cognitiva en la que se concibe el sentido de pérdida como una característica central del paciente deprimido, y donde se considera básico para la comprensión de la etiología depresiva las estructuras cognitivas negativas y las formas defectivas y primitivas de procesamiento de la información (véase también Beck, Rush, Shaw y Emery, 1979 ). Para explicar el sustrato psicológico de la depresión se utilizan tres conceptos especificos, que son: a) la tríada cognitiva, b) esquemas y c) errores cognitivos (procesamiento defectuoso de la información). Se asume que la persona deprimida tiene una visión negativa de sí misma, construye la experiencia de forma negativa y tiene una visión pesimista de su futuro a corto y largo plazo (véase Kuiper, 1978, y Rizley, 1978, donde se dan datos sobre atribuciones internas de autoculpabilización por el fracaso en sujetos clasificados como depresivos). Se piensa que estas cogniciones negativas, que en conjunto forman la tríada cognitiva, subyacen a los sintomas comunes de depresión clínica (es decir, déficits motivacionales, baja autoestima, apatia, impotencia aprendida, afecto negativo). Beck piensa, asimismo, que la persona clínicamente depresiva filtra, categoriza y evalúa las experiencias a través de una matriz de esquemas idio- 
sincráticos, prepotentes y disfuncionales, y que estos esquemas posibilitan el mantenimiento de actitudes dañosas y derrotistas, a pesar de la evidencia objetiva de que hay factores positivos en la vida del depresivo. Es más, se supone que el depresivo incurre en un procesamiento defectuoso de la información, que implica modos primitivos de organizar la realidad, tales como el pensamiento absolutista y dicotómico, la hipergeneralización y la personalización (Beck y otros, 1979, pp.14-15). Se plantea la hipótesis de que los pacientes depresivos pueden estar predispuestos, por tempranas experiencias, a formar conceptos negativos sobre sí mismos, el futuro y el mundo exterior, y que los esquemas latentes, así formados, se pueden activar con posterioridad por medio de experiencias vitales específicas. Este tipo de análisis sugiere que quienes se convierten en crónicamente deprimidos, como resultado de un desempleo prolongado, pueden incurrir en esquemas de autoculpabilización que, o bien ocultan, o personalizan las causas externas de su condición de parados. Sus actitudes negativas pueden abarcar el self, la realidad presente y el futuro anticipado, extendiéndose más allá de la situación inmediata de desempleo hacia otros muchos aspectos de sus vidas.

Desde la perspectiva de la atribución, Weiner y sus colaboradores han aportado datos que sugieren que los indicadores de depresión, tales como la apatía y la resignación, tienden a acompañar a las atribuciones del fracaso internas y estables, como cuando una persona culpa del fracaso a la falta de habilidad y a alguna otra causa interna y estable (Weiner, en prensa, Weiner, Russell y Lerman, 1978, 1979). Pero está claro que Weiner acepta también que, a menudo, la depresión puede estar ligada a adscripciones externas, como ocurre cuando una persona atribuye su estado emocional a un suceso de naturaleza catastrófica, a la pérdida de un ser querido, etc. Lo que parece importante es que debería haber una baja expectativa de lograr el objetivo, basada en causas estables. De acuerdo con Weiner (1980), entonces, la depresión puede implicar desesperanza (baja expectativa) y sentimientos negativos «que pueden generarse por adscripciones internas o externas, o por el resultado negativo de un suceso" (p.404). Esta postura es muy similar a los recientes replanteamientos de la teoría de la impotencia aprendida (Abramson, Seligman y Teasdale, 1978; Miller y Norman, 1979), excepto en que Weiner (1980) cree que la impotencia (independencia entre respuesta y resultados) es el concepto apropiado que hay que recalcar.

Abramson y otros (1978) mantienen que la depresión por impotencia ocurre cuando «se considera improbable la obtención de resultados muy deseados o probable la de resultados muy aversivos, $y$ el individuo espera que ninguna respuesta de su repertorio cambie esta probabilidad" (p.68). Describen cuatro clases de déficits que pueden ocurrir en la depresión: motivacional, cognitivo, de autoestima y afectivo. El modelo atributivo de depresión por impotencia, que ellos presentan, recurre a las dimensiones atributivas interno/externo y estable/inestable, desarrolladas anteriormente por Weiner (1978) y añaden la de global/específico como una dimensión más del proceso de atribución. Cada una de estas dimensiones juega un papel particular en relación con la depresión por impotencia. Se asume que la extensión en que los déficits depresivos se generalizan entre situaciones está relacionada con la globalidad con que se vea la causa atribuida. La cronicidad de los déficits depresivos a lo largo del tiempo está relacionada con la estabilidad de la causa atribuida. La pérdida de autoestima dependería de la internalidad de la atribución de la impotencia. Abramson y otros (1978) asumen que la «intensidad de los déficits depende de la intensidad, o certidumbre, de la expectativa de incontrolabilidad y, en el caso de los déficits afectivos y de autoestima, de la importancia del resultado" (p.68). Al igual que Weiner, reconocen que los déficits depresivos pueden estar basados, bien interna o ex- 
ternamente. De hecho, utilizan el ejemplo del desempleo para explicar su postura. Hacen la comparación de dos individuos que están deprimidos:

"porque esperan que, independientemente del esfuerzo que hagan, seguirán desempleados. La depresión de la persona que cree que su fracaso en encontrar trabajo se debe a su propia incompetencia, se vivenciará con un sentimiento de inutilidad y de poco aprecio. La persona que cree que la causa de su fracaso está en la crisis económica nacional no se considerará menos valiosa. Ambas depresiones, sin embargo, se caracterizarán por la pasividad, por cogniciones negativas y por la tristeza, que son los otros tres déficits depresivos, ya que ambos individuos tienen muy pocas esperanzas de que haya un resultado positivo, y éste no es contingente con ninguna respuesta de su repertorio" (p.66).

Estos tres análisis de la depresión y el análisis aquí presentado contienen similitudes y diferencias entre si. Los tres enfoques plantean el interés de explorar los esquemas de atribución causal que usa el parado para explicar su situación, pero, mientras que Weiner, Seligman y sus colegas toman en consideración tanto las atribuciones internas como externas, el análisis de Beck pone el acento en los esquemas de autoculpabilización entre los deprimidos. Los tres modelos son cognitivos y ponen de relieve los vínculos entre cognición y afecto, pero hay una diferencia distintiva en el énfasis cognitivo, que se relaciona con el enfoque de la expectativa-valencia y sus implicaciones. En nuestro análisis se plantea que aquellas personas que estén más motivadas a buscar trabajo, experimentarán sentimientos negativos mayores por su situación de desempleo que aquellos que estén menos motivados; es decir, ponemos de relieve la existencia de un vínculo entre la motivación positiva frustrada y los sentimientos negativos. Predecimos que, lejos de ser pasivos y resignados, los individuos que manifiesten sentimientos más depresivos expresarán mayor inte- rés en conseguir un empleo que aquellos que manifiestan un sentimiento menos depresivo. Esto es debido a que se considera que el sentimiento o afecto negativo está relacionado con una mayor expectativa de éxito, no confirmada a causa del fracaso, y/o con un mayor atractivo neto o valencia del objetivo de empleo no alcanzado. La combinación de estas dos variables se considera que determina la intensidad de la motivación para buscar trabajo. En oposición a Abramson y otros (1978), nosotros, por tanto, no postulamos un vínculo entre pasividad y depresión. ¡Al contrario! La teoría de la expectativa-valencia implica que las personas con intensa motivación para buscar trabajo, que se han visto frustradas una y otra vez en sus intentos por colocarse, expresarán sentimientos más depresivos por razón de sus fracasos que aquellas para quienes la motivación hacia el trabajo es menor. No negamos que la motivación para buscar trabajo decline con el fracaso repetido en encontrarlo. Nuestra teoría postula que esta reducción se dará conforme disminuyan las expectativas de conseguir el objetivo ${ }^{2}$. Sin embargo, afirmamos que, independientemente del declinar general de la motivación con la disminución de las expectativas, los individuos más fuertemente motivados reaccionarán a su situación de paro con un sentimiento depresivo mayor que quienes estén menos motivados para buscar trabajo.

\section{Método}

\section{Sujetos y procedimiento}

Los sujetos fueron 212 jóvenes desempleados (150 hombres, 60 mujeres, 2 sin especificar) de Adelaida, Australia del Sur. Fueron contactados a través de instituciones asistenciales relacionadas con el desempleo. Los sujetos respondieron a un cuestionario que fue administrado por el segundo autor (P.R.D.), en pequeños grupos (10-15 sujetos) durante los ameses de julio y agosto de 1978. Se tardaba unos treinta minutos en respon- 
der al cuestionario $y$, cuando estaba completo, se pagaban dos dólares a cada sujeto. La edad media de la muestra fue 19,76 años, el 96 por 100 eran solteros y el 60 por 100 dijo que vivía en casa de sus padres. El periodo medio de desempleo para la muestra era de 30,59 semanas (Mdn $=19,69$ semanas).

\section{Cuestionario}

Se dividió el cuestionario en dos secciones, una que consistía en preguntas relacionadas con la situación actual, mientras que la otra contenía preguntas de tipo retrospectivo, relacionadas con la experiencia escolar junto con algunas preguntas demográficas y de antecedentes. La mayoría de las preguntas requerían el uso de una escala de evaluación dividida en cinco partes iguales, con rótulos en cada extremo, y las respuestas a estas preguntas se puntuaron de 1 a 5 . Todas las preguntas que se describirán eran de este tipo.

Expectativa de éxito. Se diseñaron las tres preguntas siguientes para medir las expectativas de éxito presentes y futuras: (a) «QQué confianza tiene en poder conseguir un trabajo en un futuro próximo? (ninguna confianza/mucha confianza)»; (b) "¿Cuáles diría usted que son sus posibilidades de lograr un trabajo, en comparación con otra gente de su edad que está desempleada? (mayores/menores)»; y (c) "¿Qué confianza tenía en obtener un trabajo cuando dejó los estudios? (ninguna confianza/mucha confianza)».

Valencia del trabajo. Se emplearon tres preguntas para derivar una medida de la valencia o atractivo percibido del empleo: (a) «El trabajo para una persona significa algo más que sólo dinero? (no, en absoluto/sí, por supuesto)»; (b) «La mayoría de las satisfacciones en la vida de una persona provienen de su trabajo? (no, en absoluto/ sí, por supuesto)»; y (c) "¿Cuánto debe de interesarse la gente por su trabajo? (no es necesario interesarse/la gente debe de estar muy interesada)". Estos tres ítems fueron seleccionados en base a un análisis factorial que incluía el procedimiento de factor principal seguido por la rotación varimax (la solución PA/2 en Nie, Hull, Jenkins, Steinbrenner y Bent, 1975), que mostró que los ítems tenían una saturación factorial relativamente alta $(0,59$, 0,51 y 0,70 respectivamente) en un factor que hemos denominado valencia del empleo. Las medidas en estos tres ítems se sumaron para dar una medida en esta variable. Por tanto, las posibles puntuaciones para la valencia del empleo iban de 3 a 15.

Motivación para trabajar. Las siguientes preguntas se incluyeron para proveer de una medición de la motivación para buscar trabajo: (a) para el esfuerzo inicial: «¿Cómo diría usted que fue el esfuerzo que hizo para buscar trabajo inmediatamente después de terminar sus estudios? (no lo intenté/hice todo lo que pude)»; (b) para el esfuerzo actual: "¿Cómo diria usted que es el esfuerzo que hace ahora para encontrar trabajo? (no lo estoy intentando/hago todo lo que puedo)»; (c) para la necesidad inicial: "¿En qué medida quería usted un trabajo cuando acabó sus estudios? (no lo quería en absoluto/lo quería realmente)"; y (d) para la necesidad presente: «¿En qué medida siente que usted necesita un trabajo? (realmente no lo necesito/estoy desesperado por encontrar un trabajo)".

Atribuciones causales. Se pidió a los sujetos que evaluasen un conjunto de 28 razones posibles para su desempleo. Cada razón se evaluaba de acuerdo con «qué importancia cree que tiene como causa directa de que usted esté desempleado». Las razones cubrian un amplio rango: carencia de educación, falta de experiencia, carencia de habilidades específicas, poco esfuerzo, demasiada competencia, racha de mala suerte, enfermedad o accidente temporal, ser mujer, ser una persona con mala suerte, no entender inglés, ser demasiado joven para algunos trabajos, no desear un trabajo, defectos 
físicos, ser difícil de contentar, ser emigrante, apariencia pobre, incapacidad para desenvolverse bien en las entrevistas, expectativas poco realistas sobre la educación y experiencia de los que hacen la contratación, no hacer la solicitud debido a los bajos salarios pagados en muchos trabajos, no hacer la solicitud porque muchos trabajos son aburridos, grandes desplazamientos en algunos trabajos, la industria privada no ofrece suficientes puestos de trabajo, el gobierno no crea suficientes puestos de trabajo, reducción deliberada de puestos de trabajo por parte de la industria privada, efectos adversos de los sindicatos sobre el mercado de trabajo, la situación económica en Australia, rechazo a trabajar fuera de Adelaida y ser demasiado viejo para algunos trabajos. Cada una de estas razones se puntuó en una escala que iba desde no importante hasta muy importante. Además de valorar estas 28 posibles razones para el desempleo, los sujetos contestaron a la siguiente pregunta: «¿En qué medida piensa que el estar en paro es culpa suya? (no es mi culpa en absoluto/es todo culpa mía).» Nos referiremos a este ítem como atribución interna. Mientras que las otras 28 razones indicaban causas específicas, el ítem de atribución interna era bastante general y pretendia valorar el grado de autoculpabilización.

Las puntuaciones en las 28 razones y en la atribución interna se correlationaron entre sí, junto con el sexo (hombre $=1$; mujer $=2$ ) y la matriz de correlaciones producto-momento se analizó factorialmente usando la solución de factor principal en comunidades seguida de rotación varimax para factores con valores comunes mayores que 1 (la solución PA/2 en Nie y otros, 1975). Aparecieron once factores, que daban cuenta del 64,1 por 100 de la varianza total. Algunos de estos factores incluían sólo dos o tres items y se desecharon.

Dos factores rotados, sin embargo, fueron la base para las escalas derivadas. El primer factor incluía cinco ítems con saturación factorial relativamente alta: (a)
«El gobierno no está haciendo lo necesario para ayudar a crear puestos de trabajo» $(0,54)$, (b) «La industria privada está reduciendo deliberadamente el número de trabajos disponibles» $(0,44)$, (c) «Los sindicatos están afectando al desempleo, al provocar demasiadas huelgas, manteniendo muy altos los salarios, o por otras razones» $(0,60)$, (d) «La situación económica en Australia» $(0,52)$ y (e) atribución interna $(-0,37)$. A este factor se le denominó dificultad externa, ya que los items con saturación factorial positiva se referían todos a alguna condición externa que estaba causando el desempleo. Las puntuaciones en los cuatro primeros items se sumaron para dar una medida de esta variable, yendo las puntuaciones posibles de 4 a 20 .

El segundo factor incluia cuatro items con saturación factorial relativamente alta: (a) "No he conseguido una educación suficiente» $(0,51)$; (b) "No he conseguido suficiente experiencia» $(0,64)$; (c) «No he adquirido la destreza específica que muchos patrones exigen" $(0,59)$ y (d) «Hay demasiada competencia para el tipo de trabajo que yo quiero" $(0,43)$. Este factor se denominó deficiencia de competencia, ya que todos los items con saturación factorial positiva se referian a algún tipo de defecto personal en la habilidad, destreza o experiencia como causas del desempleo actual. Se sumaron las puntuaciones en los cuatro ítems para dar una medida de esta variable, yendo las puntuaciones posibles de 4 a $20^{3}$.

Sentimiento depresivo. La siguiente pregunta se incluyó para medir el sentimiento depresivo: "Cuando Vd. piensa que está desempleado ¿cómo le hace sentirse? (no todo el mundo siente lo mismo al estar en paro)"y. La escala de evaluación de 5 puntos iba desde muy contento (1) hasta muy deprimido (5). Los porcentajes de sujetos en las cinco categorías fueron: 4,7 por 100 (1), 9,5 por 100 (2), 32,2 por 100 (3), 27,5 por 100 (4) y 26,1 por 100 (5). En los análisis que siguen, se han juntado la primera $y$ segunda categorías, de forma que se pue- 
den distinguir cuatro grados de estado de ánimo: contento ( 1 y 2 ), neutral (3), deprimido (4) y muy deprimido (5).

\section{Resultados}

\section{Puntuaciones medias en relación con el} sentimiento depresivo

El cuadro 1 presenta las puntuaciones medias en las principales variables de la investigación, en relación con las cuatro categorías de estado de ánimo y los resultados de un análisis de varianza $2 \times$ 4 , con el sexo (hombre, mujer) como primer factor, y estado de ánimo (contento, neutral, deprimido y muy deprimido) como segundo factor.

El cuadro 1 muestra que las diferencias de estado de ánimo entre contento y muy deprimido estaban asociadas con incrementos estadísticamente significativos en la confianza inicial, valencia del em- pleo, esfuerzo actual, necesidad inicial, necesidad actual y dificultad externa, y con una disminución significativa en la atribución interna. Es decir, los sujetos con mayores niveles de sentimiento depresivo fueron los que indicaron que al principio estaban más confiados en encontrar un trabajo y con más necesidad de ello al terminar los estudios, que veían el empleo como un objetivo atractivo, que indicaban altos niveles de esfuerzo y necesidad. en ese momento y que tendian a culpar de su situación de parados, no a ellos mismos, sino a dificultades externas relativamente estables. Las diferentes clasificaciones de estado de ánimo estaban también asociadas con diferencias estadísticamente significativas en la estimación de las posibilidades actuales de encontrar un trabajo, pero las relaciones no eran lineales y no trataremos más sobre ello.

No habia diferencias estadísticamente

CuAdro 1. Puntuaciones medias de las principales variables con relación a las cuatro categorias de estado de ánimo

\begin{tabular}{|c|c|c|c|c|c|c|}
\hline \multirow[b]{2}{*}{ Variable } & \multirow[b]{2}{*}{ Rango } & \multicolumn{4}{|c|}{ Categoria de estado de ánimo } & \multirow{2}{*}{$\begin{array}{l}\text { Efecto } \\
\text { principal } \\
\text { del sen- } \\
\text { timiento } \\
\text { depresivo } \\
\mathrm{F}(3,201)\end{array}$} \\
\hline & & $\begin{array}{l}\text { Con- } \\
\text { tento } \\
(n=30)\end{array}$ & $\begin{array}{l}\text { Neutral } \\
(n=68)\end{array}$ & $\begin{array}{l}\text { Depri- } \\
\text { mido } \\
(\mathrm{n}=58)\end{array}$ & $\begin{array}{l}\text { Muy de- } \\
\text { primido } \\
(\mathrm{n}=55)\end{array}$ & \\
\hline \multicolumn{7}{|l|}{ Expectativa de éxito } \\
\hline Confianza inicial & $1-5$ & 3,07 & 4,01 & 4,26 & 4,30 & $7,38 * * *$ \\
\hline Posibilidades actuales & $1-5$ & 2,53 & 3,15 & 2,93 & 2,85 & $3,02 *$ \\
\hline Confianza actual & $1-5$ & 3,03 & 3,18 & 3,14 & 3,09 & 0,03 \\
\hline $\begin{array}{l}\text { Valencia del empleo } \\
\text { Motivación para trabajar }\end{array}$ & $3-15$ & 9,77 & 11,03 & 11,19 & 11,76 & $3,47^{*}$ \\
\hline Esfuerzo inicial & $1-5$ & 3,47 & 3,93 & 4,02 & 4,31 & 2,32 \\
\hline Esfuerzo actual & $1-5$ & 2,47 & 3,21 & 3,24 & 3,62 & $5,06 * *$ \\
\hline Necesidad inicial & $1-5$ & 3,27 & 4,09 & 4,42 & 4,46 & $7,17 * * *$ \\
\hline $\begin{array}{l}\text { Necesidad actual } \\
\text { Atribución causal }\end{array}$ & $1-5$ & 2,20 & 3,32 & 4,07 & 4,15 & $25,17 * * *$ \\
\hline $\begin{array}{l}\text { Atribución interna } \\
\text { Deficiencia en la competen- }\end{array}$ & $1-5$ & 3,40 & 2,51 & 2,19 & 2,22 & $7,05 * * *$ \\
\hline cia & $4-20$ & 13,67 & 12,40 & 14,17 & 13,00 & 2,44 \\
\hline Dificultad externa & 4-20 & 12,27 & 13,53 & 14,60 & 14,45 & $2,80^{*}$ \\
\hline
\end{tabular}

Nota: Hubo pequeñas variaciones en $n$ debido a la pérdida de datos en algunos análisis (1-3 casos).

${ }^{*} \mathrm{p}<0,05 .{ }^{* *} \mathrm{p}<0,01$.** $\mathrm{p}<0,001$. 
significativas para el sexo en ninguna de las variables indicadas en el cuadro 1 , incluido el sentimiento depresivo. El único efecto interactivo significativo se dió con las puntuaciones de esfuerzo actual, $F$ $(3,201)=3,49, \mathrm{p}<0,05$. El incremento de niveles bajos a altos en las puntuaciones medias del esfuerzo de ese momento, a través de las cuatro categorías (contento a muy deprimido), era más pronunciado entre los sujetos femeninos.

\section{Correlaciones entre las principales variables}

La estructura de las relaciones entre variables se expone en el cuadro 2, que presenta las correlaciones producto-momento entre todas las variables principales.

El cuadro 2 muestra que había relaciones positivas estadísticamente significativas entre sentimiento depresivo y confianza inicial, valencia del empleo, esfuerzo inicial, esfuerzo actual, necesidad inicial, necesidad actual y dificultad externa. Había también una correlación negativa estadisticamente significativa entre sentimiento depresivo y atribución interna. Todos estos resultados son consistentes con los señalados en el cuadro 1 . Añaden información sobre la intensidad y la estructura de las relaciones.

$\mathrm{El}$ cuadro 2 indica asimismo que otra serie de correlaciones importantes lograron la significación estadística. Como era de esperar, las mediciones de la misma variable estaban correlacionadas positivamente (p. ej., confianza actual con posibilidades actuales, esfuerzo inicial con esfuerzo actual, necesidad inicial con necesidad actual). La atribución interna estaba relacionada negativamente con la confianza inicial, y la dificultad externa estaba positivamente relacionada con la confianza inicial. Así, los sujetos que indicaron que al principio estaban relativamente confiados en encontrar un trabajo eran más propensos a atribuir su desempleo a dificultades externas (tales como la situación económica, la inactividad del gobierno, la política deliberada de la industria privada y los efectos negativos de los sindicatos) y menos propensos a culparse a sí mismos, en comparación con los sujetos cuyas expectativas iniciales de empleo eran más bajas. La deficiencia de competencia (carencia de educación, experiencia, habilidades específicas, etc.) no estaba implicada en ninguna relación significativa con las otras variables.

Las medias en el cuadro 2 señalan que las puntuaciones de confianza, esfuerzo y necesidad tendían a ser menores en la situación presente en comparación con la situación al terminar sus estudios. Las tres diferencias de medias eran estadísticamente significativas. Para la confian$\mathrm{za}, \mathrm{t}(208)=7,52, \mathrm{p}<0,001$; para $\mathrm{el}$ esfuerzo, $\mathrm{t}(211)=6,76, \mathrm{p}<0,001 ; \mathrm{y}$ para la necesidad, t (208), $\mathrm{p}<0,001$. Estas disminuciones son consistentes con nuestro presupuesto de que el fracaso repetido en conseguir un trabajo lleva a una disminución de las expectativas de éxito y de los niveles de motivación.

Es interesante anotar, sin embargo, que las mediciones de expectativa, valencia, motivación para trabajar y sentimiento depresivo, no tenían una relación significativa con las declaraciones de los sujetos sobre las semanas que llevaban sin trabajar. La duración del desempleo, no obstante, estaba relacionada negativamente, tanto con la medición del esfuerzo inicial, $\mathrm{r}(210)=-0,14, \mathrm{p}<0,05$ (es decir, mayor tiempo en paro, menores puntuaciones de esfuerzo inicial), cuanto con la apreciación de que las cualificaciones escolares eran mayores en comparación con las de otras personas de la misma edad que estaban buscando trabajo, $\mathrm{r}(210)=-0,15, \mathrm{p}<0,05$ (es decir, mayor tiempo de desempleo/menores puntuaciones en la calificación escolar). El tiempo de desempleo estaba positivamente relacionado con la medida de dificultad externa, $\mathrm{r}(210)=0,14, \mathrm{p}$ $<0,05$ (es decir, mayor tiempo de desempleo/mayores puntuaciones de dificultad externa) ${ }^{4}$. 
CUADRO 2. Correlaciones producto-momento entre las principales variables, junto con las medias y desviaciones tipicas

Variable

\begin{tabular}{|c|c|c|c|c|c|c|c|c|c|c|c|c|c|c|}
\hline Variable & 2 & 3 & 4 & 5 & 6 & 7 & 8 & 9 & 10 & 11 & 12 & 13 & M & $\sigma$ \\
\hline 1 Confianza inicial & $0,16^{*}$ & 0,09 & 0,07 & 0,13 & $0,15^{*}$ & $0,53 * * *$ & $0,19 * *$ & $-0,29^{* * *}$ & $-0,04$ & $0,21 * *$ & 0,06 & $0,29 * * *$ & 4,00 & 1,30 \\
\hline 2 Posibilidades actuales & & $0,28 * * *$ & $0,23 * *$ & 0,01 & 0,03 & 0,07 & $0,19 * *$ & $-0,07$ & $-0,09$ & 0,05 & 0,01 & 0,07 & 2.92 & 0,94 \\
\hline 3 Confianza actual & & & $0,22 * *$ & $-0,08$ & $0,14 *$ & 0,05 & $0,19 * *$ & 0,00 & $-0,10$ & $-0,03$ & $0,14^{*}$ & 0,01 & 3,13 & 1,20 \\
\hline 4 Valencia del empleo & & & & 0,13 & 0,12 & 0,12 & $0,18^{*}$ & $-0,01$ & 0,03 & 0,11 & $0,15^{*}$ & $0,23 * *$ & 11,10 & 2,69 \\
\hline 5 Esfuerzo inicial & & & & & $0,23 * *$ & $0,30^{* * *}$ & $0,15 *$ & $-0,09$ & 0,08 & 0,01 & 0,10 & $0,19 * *$ & 3,99 & 1,35 \\
\hline 6 Esfuerzo actual & & & & & & 0,13 & $0,30 * * *$ & $-0,13$ & $-0,11$ & 0,06 & 0,06 & $0,25 * * *$ & 3,22 & 1,35 \\
\hline 7 Necesidad inicial & & & & & & & $0,31 * * *$ & $-0,29 * * *$ & 0,09 & $0,14^{*}$ & 0,08 & $0,29 * * *$ & 4,16 & 1,27 \\
\hline 8 Necesidad actual & & & & & & & & $-0,32 * * *$ & 0,06 & $0,16^{*}$ & 0,05 & $0,50 * * *$ & 3,57 & 1,28 \\
\hline 9 Atribución interna & & & & & & & & & 0,03 & $-0,17^{*}$ & $-0,02$ & $-0,29 * * *$ & 2,49 & 1,32 \\
\hline 10 Deficiencia en la compe- & & & & & & & & . & & 0,12 & 0,09 & & 13,24 & 3,83 \\
\hline 11 Dificultad externa & & & & & & & & . & & 0,12 & $-0,03$ & $0,20 * *$ & 13,84 & 4,08 \\
\hline 12 Sexo & & & & & & & & & & & & 0,09 & 1,29 & 0,45 \\
\hline 13 Sentimiento depresivo & & & & & & & & & & & & & 3,61 & 1,11 \\
\hline
\end{tabular}

Nota: $\mathrm{n}$ va desde 209 hasta 212, debido a la pérdida de datos. Se usaron pruebas de significación de dos colas.

* $\mathrm{p}<0,05$. ** $\mathrm{p}<0,01$. *** $\mathrm{p}<0,001$. 


\section{Discusión}

Los resultados muestran que el sentimiento depresivo en esta muestra de jóvenes desempleados tendía a estar asociado con los niveles más altos de motivación para buscar trabajo y con esquemas de atribución causal que absolvían de culpa a uno mismo y daban mayor importancia a las dificultadés externas como factores causales. De esta forma, los jóvenes desempleados con mayores niveles de sentimiento depresivo eran más propensos a señalar que necesitaban un trabajo, que habían intentado encontrarlo, y que no se culpaban a sí mismos por su situación de parados, en comparación con los jóvenes desempleados con menores niveles de sentimiento depresivo. Tendian, asimismo, a dar mayores niveles de confianza inicial en la obtención de un trabajo y a considerar el trabajo de forma más atractiva (valencia positiva), e inclinados a considerar que ciertos factores externos, relativamente estables, eran el origen de sus actuales dificultades.

Este tipo de resultados es consistente con las implicaciones de un análisis de la expectativa-valencia, que presupone que la motivación positiva o tendencia a buscar empleo está relacionada con la combinación multiplicativa de expectativa de éxito y atractivo neto percibido (o valencia). También lo son las disminuciones observadas en los niveles de expectativa, esfuerzo y motivación desde el abandono de la escuela hasta el momento actual, disminuciones que se pueden relacionar con el fracaso en encontrar un empleo estable, a pesar de los intentos realizados durante un período de tiempo considerable (30 semanas, como media). Un análisis de expectativa-valencia predice que el fracaso repetido en encontrar un trabajo determinará expectativas reducidas de éxito y una disminución en la tendencia a buscar empleo, suponiendo que las causas del fracaso se van a considerar como relativamente estables $e$ inmodificables ${ }^{5}$.

Nótese, sin embargo, que los resulta- dos no son consistentes con una visión de la impotencia aprendida, que implica que los individuos que señalan altos niveles de sentimiento depresivo serán más pasivos y estarán menos motivados.

El esquema de atribución causal de los sujetos de este estudio que expresaron mayores niveles de sentimiento depresivo, implicaba una atribución interna menor y una atribución relativamente más externa, en comparación con los individuos que expresaron menores niveles de sentimiento depresivo. Estas atribuciones tienen su propia lógica inherente. Los individuos con mayores niveles de sentimiento depresivo tendian también a expresar mayores niveles de necesidad y de confianza inicial. Se podía entonces esperar que fuesen menos propensos a ver su actual situación negativa como resultante de falta de motivación (un factor interno). En su lugar, se espera que recurran más a razones externas, tal como la idea ampliamente difundida de que el desempleo actual es producto de fuerzas sociales, políticas y económicas, constantemente reforzada por los medios masivos de comunicación. Tal información consensuada (Kelley, 1973) proveería de una base para atribuir los fracasos a dificultades externas. Las atribuciones internas que implican autoculpabilización serían más comunes entre los jóvenes desempleados con menor confianza, que se sentirían no suficientemente motivados para ponerse a buscar trabajo. Sería más fácil para estas personas el recurrir a factores internos, tales como falta de motivación, como causas de su fracaso para obtener un trabajo. Estas personas menos motivadas serian también las que tenderian a expresar menores niveles de estado de ánimo negativo a causa de su situación de parados.

\section{Algunas matizaciones}

Así, estos datos constituyen un conjunto coherente y significativo, pero hay que hacer cuatro matizaciones. La primera es que los datos se basan en autoinformes, algunos de carácter retrospectivo. Para que las conclusiones adquieran 


\section{Estudios}

firmeza tendrian que haberse incluido medidas conductuales (p. ej., observaciones de la conducta actual de búsqueda de trabajo). Por desgracia, tales medidas son dificiles de obtener, y no pudimos hacerlas. Es posible, por tanto, que los resultados reflejen teorías implícitas que los sujetos tienen sobre el sentimiento depresivo y el desempleo, aunque no pensamos que éste sea el caso. No tenemos razones para dudar de que quienes respondieron diciendo que estaban desesperados por encontrar un trabajo no estuviesen de hecho buscándolo activamente, pero para estar seguro en este punto se necesitan medidas conductuales objetivas.

En algunos estudios recientes, que tra$\tan$ la forma en que la gente se ajusta al cambio económico, se ha investigado la incidencia de posibles consecuencias, tales como empeoramiento de la salud, desórdenes físicos y emocionales, relaciones sociales defectuosas e intentos de suicidio, entre los que padecen condiciones económicas adversas, que les llevan al despido y al desempleo (p. ej., Brenner, 1973; Dooley y Catalano, 1980; Ferman y Gordus, 1979). Estos estudios indican cómo se puede ir más allá de los informes verbales en futuros estudios sobre los efectos del desempleo. De todas formas, la medida del informe verbal sobre el sentimiento depresivo utilizada en este estudio estaba inmersa en un conjunto de resultados coherentes y teóricamente llenos de sentido.

En segundo lugar, los resultados están relacionados con nuestra muestra, compuesta por jóvenes desempleados en contacto con instituciones asistenciales. Es gente que aún busca oportunidades y apoyo y que, presumiblemente, mantienen una cierta esperanza de que su situación pueda mejorar. Si hubiéramos podido preguntar a jóvenes desempleados que han abandonado la búsqueda de empleo, podríamos haber encontrado que en ciertos aspectos diferían en las variables que se midieron, con respecto a la muestra utilizada. De forma general, podrian haber sido más pasivos, más incli- nados a atribuir sus fracasos a deficiencias personales, más bajos en autoestima, mucho más pesimistas en sus expectativas y más firmemente convencidos de que las circunstancias no cambiarian, con un sentimiento depresivo más intenso y generalizado a otras muchas áreas de su vida. Pero el problema está en encontrar estos jóvenes desempleados que ya no compiten por el trabajo. Nuestro procedimiento de muestreo no permitía captarlos.

En tercer lugar, hubiera sido muy ventajoso obtener datos de un estudio longitudinal, de forma que se pudiesen observar los cambios en las expectativas, niveles de motivación, estado de ánimo y atribuciones a lo largo del tiempo para el mismo grupo de sujetos. Se estaría entonces en una posición más fuerte para poder hacer afirmaciones sobre los antecedentes causales. No obstante, los datos recogidos en un momento dado pueden ser también relevantes para probar predicciones derivadas teóricamente, como era el caso del presente estudio.

En cuarto lugar, la medida del sentimiento o estado de ánimo depresivo usada en este estudio era una medida específica para una situación, relativa al estado de ánimo depresivo asociado con la condición de parado. Nuestro estudio se refería a formas crónicas de depresión, que se generalizan entre situaciones, y que se han evaluado con mediciones tales como el Beck Depression Inventory (Beck, 1967). El tema de la medición de la depresión es complejo (Depue y Monroe, 1978) y escapa a los fines de esta discusión, pero es posible que si hubiésemos medido la depresión crónica entre los parados, hubiésemos descubierto datos sobre esquemas de autoculpabilización y de otras cogniciones negativas (Beck y otros, 1979), autoeficacia disminuida (Bandura, 1977a), atribuciones caracterológicas (Janoff-Bulman, 1979) y estilos atributivos que suponen atribuciones del fracaso internas, estables y globales (Abramson y otros, 1978; Seligman, Abramson, Sommel y von Baeyer, 1979). De nuevo, sin embargo, el pro- 


\section{Estudios}

blema está en localizar a estos depresivos crónicos con la red de muestreo.

\section{Efectos del desempleo prolongado}

Este último punto pone de manifiesto un tema interesante que se relaciona con el presente modelo. En la revisión que Eisenberg y Lazarsfeld (1938) hicieron sobre los efectos psicológicos del desempleo en los años 30 , se describen una serie de reacciones adversas, que incluyen la apatía, resignación, depresión, dudas sobre sí mismo, disminución de la autoestima y creencias fatalistas. Aunque la situación de desempleo en los años 70 y 80 difiere en algunos aspectos de la de los años 30 (Jahoda, 1979), podría esperarse aún encontrar que el desempleo prolongado estuviera asociado con altos niveles de depresión general entre algunas personas. ¿Son inconsistentes con esta expectativa las implicaciones del análisis de la expectativa-valencia? Nuestro modelo implica que el desempleo prolongado determina una disminución de las expectativas y una reducción de la motivación para buscar trabajo, reflejado en conductas tales como apatía y resignación. Sin embargo, también implica que el sentimiento depresivo disminuye conforme las perspectivas de encontrar un trabajo se hacen remotas y desaparece la motivación para buscar un empleo (es decir, menos motivación/menos sentimiento depresivo). Esta última implicación debería, sin embargo, situarse dentro de un contexto más amplio que tuviese en consideración la necesidad de distinguir entre depresión específica de una situación y otras formas de depresión más generales y crónicas. De esta manera, podria haber personas que se vuelven crónicamente deprimidas a raíz de un paro prolongado, ya que estaban predispuestas, por experiencias previas, a desarrollar desórdenes depresivos (Beck y otros, 1979). Es más, dado que el desempleo prolongado puede tener efectos devastadores en muchos aspectos de la vida de una persona, podría esperarse que las reacciones depresivas se relacio- nasen con el ámbito total de las consecuencias negativas y no sólo con la frustrada motivación para el trabajo. Un nivel alto de sentimiento depresivo puede, por tanto, reflejar la suma de muchos malestares distinguibles pero interconectados. Finalmente, es posible que el desempleo prolongado, para algunas personas, dé como resultado un cambio cualitativo en las atribuciones causales, de forma que se hagan más frecuentes las atribuciones a factores internos, globales, estables e incontrolables, a pesar de la evidencia de que muchas de las causas del desempleo son externas y socioeconómicas. Es decir, se puede dudar de la autoeficacia en conseguir un trabajo cuando los niveles de pesimismo son altos. El desempleo prolongado puede, por tanto, tener efecto no sólo en las expectativas de resultados (como se ha argumentado), sino también en las expectativas de eficacia (Bandura, 1977a) y en las creencias acerca de las condiciones causales subyacentes (para una discusión sobre estos tipos diferentes de expectativas, véase Heckhausen, 1977). Las formas crónicas de depresión pueden desarrollarse como resultado de algunos de estos efectos (véase la discusión anterior sobre las ideas teóricas de Abramson y otros, 1978; Beck y otros, 1979, y Weiner, 1980).

Nuestro análisis motivacional debería verse en relación con este contexto total de efectos posibles. No niega que el desempleo prolongado pueda estar asociado en algunas personas con un conjunto complejo de reacciones cognitivas, motivacionales y afectivas o con elevados niveles de depresión crónica. Lo que mantiene, sin embargo, es que uno de los factores que contribuyen a generar el estado de ánimo depresivo, a raíz de los intentos sin éxito por obtener trabajo, es la frustración de la motivación para trabajar.

\section{Depresión y atribuciones internas/externas}

En el presente estudio, los niveles más altos de sentimiento depresivo están asociados con una tendencia más débil a 
culparse a si mismo por el desempleo y con un mayor énfasis en las dificultades externas como factores causales. Un estudio reciente de Peterson, Schwartz y Seligman (en prensa) ha mostrado, sin embargo, que las atribuciones externas para los malos acontecimientos parecen ser incompatibles con sintomas depresivos. En su estudio, los síntomas depresivos tendian a vincularse con atribuciones caracterológicas para los malos acontecimientos, es decir, con atribuciones referidas a características incontrolables y recurrentemente estables del carácter de una persona. Las atribuciones externas para los malos acontecimientos estaban relacionadas negativamente con los sintomas depresivos, como eran las atribuciones conductuales en las que la culpa se dirigia a la propia conducta (véase Janoff-Bulman, 1979, para una discusión sobre la distinción entre autoculpabilización caracterológica y conductual). Peterson $\mathrm{y}$ otros (en prensa) han utilizado el Beck Depression Inventory (Beck, 1967) para medir el grado de depresión. Ya hemos hecho notar que esta medida tiene que ver con formas más generales de depresión, en contraste con las medidas más especificas de sentimiento depresivo usadas en este estudio. Las diferencias en los resultados de uno y otro estudio pueden, por tanto, deberse a las diferentes formas de depresión evaluadas.

En términos generales, sin embargo, creemos que sería prematuro concluir que la atribución externa es incompatible con los sintomas depresivos. Como se ha indicado antes, Abramson y otros (1978) reconocen que los déficits depresivos pueden estar basados interna o externamente, e ilustran este punto refiriéndose a las atribuciones causales para el desempleo. Las causas externas a las que se refieren los depresivos en sus atribuciones de los sucesos negativos pueden ser tan estables e incontrolables como las causas de base caracterológica. Es interesante notar aquí que en el estudio de Peterson y otros, las causas externas para los acontecimientos adversos fueron las más altamente puntuadas en incontrolabilidad. En este contexto es plausible argumentar que una persona desempleada, que sufre de depresión general, puede atribuir una gran parte de culpa al adverso clima económico, al que concibe como una condición relativamente estable e incontrolable, en el sentido de que la mayoría de las personas no tienen poder para cambiarlo, dado el hecho de que el desempleo está muy extendido, y es explicado, con frecuencia, en términos de fuerzas que trascienden al individuo. Probablemente haya, pues, unas condiciones en que las atribuciones a causas externas, estables e incontrolables estén presentes en la sintomatología depresiva, además de la autoculpabilización caracterológica. Es obvio, de todas formas, que se requiere una investigación sobre esta compleja cuestión.

\section{Implicaciones generales}

¿Cuáles son las implicaciones generales de los resultados que hemos aportado y del enfoque teórico que ha provisto de un marco de referencia para su interpretación? Ya hemos hecho notar una implicación, el que estos resultados no parecen ser consistentes con una teoria de los sentimientos depresivos y pasividad. Wortman y Dintzer (1978), en una amplia discusión de la teoría reformulada de la impotencia aprendida en los seres humanos (Abramson y otros, 1978), indican que el nuevo modelo no es muy específico respecto a la relación entre los componentes motivacionales, cognitivos $y$ afectivos de la impotencia, aunque se asume que un déficit en estos tres componentes, así como en la autoestima (asumiendo la atribución interna), constituyen un sindrome asociado con la depresión de impotencia. El presente análisis teórico, junto con los resultados empíricos, ayuda a clarificar la relación entre variables motivacionales y afectivas, pero lo hace de forma que se cuestiona el presupuesto de que el estado de ánimo depresivo implica carencia de motivación.

Wortman y Dintzer (1978) señalan, 
asimismo, que otras variables distintas de las discutidas por Abramson y otros, pueden influenciar las reacciones hacia resultados incontrolables. Lo mismo hace Roth (1980) en un reciente articulo en el que resume una serie de variables que se espera influya en la impotencia aprendida en los humanos, entre las cuales están las expectativas de independencia entre respuesta y esfuerzo y el valor de la ejecución exitosa. Su análisis parece congeniar con las ideas de la expectativavalencia, ya que esta autora adopta como premisa básica de su modelo la propuesta de que ues más probable que las personas actúen sobre su medio cuando los valores de la recompensa potencial sean más elevados y lo sea también la probabilidad percibida de lograrla» (p.115).

Estas discusiones son útiles en el sentido de que plantean algunos problemas no resueltos en el modelo reformulado de la impotencia aprendida en los seres humanos y abren la posibilidad de conceptualizaciones que van más allá de la teoría de la atribución y de las expectativas de incontrolabilidad. En nuestra opinión, sin embargo, gran parte de lo escrito sobre el tema se resiente de un fallo a la hora de unir el análisis con un modelo motivacional explícito. Nosotros sugerimos que la teoría motivacional basada en los enfoques de la expectativavalencia puede hacer ciertas aportaciones en los muy dificiles temas implicados en este área de la impotencia y la depresión. Existen, por ejemplo, una gran cantidad de escritos acumulativos sobre la motivación de logro que emplean conceptos de expectativa-valencia y que aportan análisis relativamente sofisticados sobre las relaciones entre motivación, adopción de riesgos, persistencia, latencia y ejecución (Atkinson, en prensa; Atkinson y Birch, 1978; Atkinson y Feather, 1966; Atkinson y Raynor, 1974; Feather, en prensa a). Estos trabajos han sido más bien ignorados por parte de los investigadores de la impotencia aprendida, aunque tienen implicaciones para algunos de los fenómenos no cubiertos por estos investigadores (p. ej., efectos de fa- cilitación y disminución de ejecución; véase Roth, 1980; Wortman y Brehm, 1975; Wortman y Dintzer, 1978).

Una de las implicaciones de la teoría de la expectativa-valencia, tal como se ha utilizado en este artículo, es que las variables que influencian las expectativas de una persona y la valencia neta del logro de un objetivo deberían relacionarse con el malestar y el estado de ánimo depresivo que puede seguirse del fracaso en el logro del objetivo. Será suficiente con un ejemplo. Se puede presuponer que los motivos y valores dominantes en una persona influirán en la valencia de las actividades particulares y de los posibles resultados, tal como se perciben en un momento dado (Feather, en prensa, a, d). Se podría aceptar, por ejemplo, que el trabajo tiene una mayor valencia positiva para aquellos individuos con fuertes valores de la ética protestante que para quienes estos valores son más débiles. Si esto es válido, se puede concluir que las personas con fuertes valores de la ética protestante serán más persistentes en sus esfuerzos por obtener un empleo y padecerán unos estados de ánimo más negativos si fracasan en la obtención del trabajo. Este tipo de implicaciones se derivan del enfoque de la expectativa-valencia, y muchas de ellas están aún por verificar.

Por último, es necesario que se hagan análisis más precisos de las condiciones bajo las cuales se dan los diferentes tipos de estado de ánimo a raiz del fracaso (p. ej., Blaney, 1977, p.210; Pittman y Pittman, 1979). ¿Los sentimientos que se dan tras el fracaso siguen algún tipo de secuencia regular o pueden darse simultáneamente, de forma que, por ejemplo, uno pueda estar tanto enfadado como deprimido, o ansioso y hostil, o ansioso y deprimido? Si aceptamos que el desempleo crónico puede llevar no sólo a la depresión sino también a sentimientos de resentimiento y hostilidad, ¿bajo qué condiciones será uno de ellos el sentimiento predominante? ¿La depresión que sigue al desempleo es diferente de la que se da a raiz de otras privaciones y pérdidas? ¿Cómo influyen las características 
de personalidad y las condiciones situacionales en el tipo de estado de ánimo experimentado? ¿Qué papel juegan las atribuciones causales? ¿Hay algunos tipos de frustración y pérdida que conducen de forma más predictible a la depresión y/o a la hostilidad que otros? Esta clase de preguntas esperan una respuesta, tanto teórica como empírica. No son cuestiones del todo nuevas. Existen bastantes escritos sobre temas tales como los efectos de la frustración, el etiquetaje de emociones, las consecuencias afectivas del fracaso en situaciones de logro, la naturaleza del stress, teorías de la conducta y experiencia emocional, psicopatología del afecto y el papel de las atribuciones causales en la experiencia afec- tiva; escritos que son relevantes para estas preguntas y que suponen un primer paso.

El presente artículo ha hecho una contribución a algunos de estos temas al indicar la posibilidad de abordarlos desde el punto de vista de la teoría de la expectativa-valencia, vinculada con ideas de la teoría de la atribución. Al margen de la contribución teórica general, el análisis ayuda a llenar una laguna, sugiriendo un marco de análisis para el estudio de alguno de los efectos psicológicos del desempleo. Supone, por tanto, una aportación para el fortalecimiento teórico de un problema social importante, que ha sido relativamente ignorado por los psicólogos.

\section{Notas bibliográficas}

1 Gurney, R. M. y Taylor, K. F. Research on the piycbology of unemployment: Some criticisms and suggestions. Melbourne Psychology Reports, n. ${ }^{\circ}$ 60, 1979. (Disponible a través de K. F. Taylor. Department of Psychology, University of Melbourne, Parkville, Victoria, Australia 3052.)

2 Linsenmeier, J. A. W. y Brickman, P. Expectations, perfomance, and satisfaction. Manuscrito no publicado. University of Michigan, 1980.

1 Beck (1976) relaciona también la tristeza y formas más externas de depresión con la experiencia de pérdida.

2 Un fracaso repetido puede también llevar a una revaluación del objetivo, de forma que se le perciba como menos atractivo (p. ej., puede reducirse la importancia del trabajo) y al descubrimiento, tanto de caracteristicas negativas propias de la búsqueda del trabajo (p. ej., entrevistas agotadoras), cuanto de obstáculos que no se habian previsto (p. ej., falta de habilidad para presentarse adecuadamente en las entrevistas). Todos estos factores reducirian el afăn positivo.

${ }^{3}$ El ítem de atribución interna tenía una saturación de 0,09 en el factor de deficiencia de competencia. Nótese también que las respuestas tendian hacia el polo uno es en absoluto culpa mía» de la escala ( $\mathrm{M}=$ $2,49)$ y que cl ítem tenía una saturación de $-0,37$ en el factor de dificultad externa. Así, aunque el ítem de atribución interna fue diseñado para poner de manifiesto el grado de autoculpabilización general, parece que muchos sujetos lo usan para indicar que no se culpan a sí mismos por su situación pero que, por implicación, culpaban a factores externos.

4 El tiempo de desempleo indicado por los sujetos es una medida muy general que no tiene en cuenta los detalles de conducta y experiencia a lo largo del tiempo. Es claro, desde el punto de vista de la expectativa-valencia que, cuando se trata de los cambios en las expectativas y motivaciones, necesitamos un análisis más detallado de variables tales como el número y estructura de los intentos no exitosos para encontrar trabajo y la forma en que los resultados negativos son interpretados por quienes buscan trabajo. Son necesarios, obviamente, estudios longitudinales del desempleado para poder poner de manifiesto los efectos causales y clarificar los detalles de los acontecimientos vitales.

5 Nótese de nuevo que otros factores pueden determinar la disminución de los niveles de motivación, conforme al enfoque de la expectativa-valencia (véase la nota 2). Nótese asimismo que las fuentes de motivación persistentes e insatisfechas se pueden acumular, de acuerdo con la teoria de la motivación de logro (Weiner, 1980), fracaso que tendria unos efectos tanto cognitivos como motivacionales (véase también Atkinson y Birch, 1978). Nosotros asumimos, sin embargo, que la motivación para buscar trabajo tenderá a decrecer debido a los intentos repetidos e infructuosos de obtener un empleo. 


\section{Referencias}

Abramson, L. Y., Sfeligman, M. E. P. y Teasdale, J. D. Learned helplessness in humans: Critique and reformulation. Journal of Abnormal Psychology, 1978, 87, 49-74.

ATKINSON, J. W. Old and new conceptions of how expected consequences influence actions. En N. T. Feather (Ed.) Expectations and actions: Expectancy-value models in psychology. Hillsdale, N. J.: Erlbaum, en prensa.

AtKinson, J. W. y Birch, D. An introduction to motivation. New York: Van Nostrand Reinhold, 1978.

AtKinson, J. W. y FEather, N. T. (Eds.) A theory of achievement motivation. New York: Wiley. 1966.

ATKINSON, J. W. y RAYNOR, J. O. (Eds.) Motivation and achievement. Washington, D.C.: Winston, 1974.

BANDURA, A. Self-efficacy: Toward an unifying theory of behavioral change. Psycbological Review, 1977, 84, $191-215(\mathrm{a})$.

Bandura, A. Social learning theory. Englewood Cliffs, N. J.: Prentice-Hall, 1977 (b).

Bandur A, A. The self system in reciprocal determinism. American Psycbologist, 1978, 33, 344-358.

BECK, A. T. Depression: Clinical, experimental and theoretical aspects. New York: Harper and Row, 1967.

BECK, A. T. Cognitive therapy and the emotional disorders. New York: International Universities Press, 1976.

Beck, A. T., Rush, A. J., Shaw, B. F. y Emery, G. Cognitive therapy of depression. New York: Guilford Press, 1979.

BLANEY, P. H. Contemporary theories of depression: Critique and comparison. Journal of Abnormal Psychology, 1977, 86, 203-233.

Brenner, M. H. Mental illness and the economy. Cambridge, Mass.: Harvard University Press, 1973.

DFPUE, R. A. y MONROE, S. M. Learned helplessness in the perspective of the depressive disorders: Conceptual and definitional issues. Journal of Abnormal Psychology, 1978, 87, 3-20.

DOOLFY, D. y CATALANO, R. Economic change as a cause of behavioral disorders. Psychological Bulletin, $1980,87,450-468$.

Eisenberg, P. y LAZARSfeld, P. F. The psychological effects of unemployment. Psychological Bulletin, $1938,35,358-390$.

Feather, N. T. Subjective probability and decision under uncertainty. Psycbological Review, 1959, 66, $150-164$.

FEATHER, N. T. Mowrer's revised two-factor theory and the motive-expectancy-value model. Psychological Review, 1963, 70, 500-515.

FEATHER, N. T. Attribution of responsability and valence of success and failure in relation to initial confidence and task perfomance. Journal of Personality and Social Psychology, 1969, 13, 129-144. .

FEATHER, N. T. Actions in relation to expected consequences: An overview of a research program. En N. T. Feather (Ed.) Expectations and actions: Expectancy-value models in psychology. Hillsdale, N. J.: Erlbaum, en prensa (a).

FEATHER, N. T. Expectancy-value approaches: Present status and future directions. En N. T. Feather (Ed.) Expectations and actions: Expectancy-value models in psychology, Hillsdale, N. J.: Erlbaum, en prensa (b).

FEATHER, N. T. (Ed.) Expectations and actions: Expectancy-value models in psychology. Hillsdale, N. J.: Erlbaum, en prensa (c).

FEATHER, N. T. Human values and the production of action: An expectancy-valence analysis. En N. T. Feather (Ed.) Expectations and actions: Expectancy-value models in psycbology, Hillsdale, N. J.: Erlbaum, en prensa (d).

Ferman, L. y Gordus, J. (Eds.) Mental bealth and the economy. Kalamazoo, Mich.: Upjohn Foundation, 1979.

HARRISON, R. The demoralising experience of prolonged unemployment. Department of Employment Gaqette, 1976, 84, 339-340.

Hartley, J. Psychological approaches to unemployment. Bulletin of the British Psychological Society, 1980, $33,412-414$.

HECKHAUSEN, $\mathrm{H}$. Achievement motivation and its constructs: A cognitive model. Motivation and Emotion, 1977, 1, 283-329.

HYMAN, H. H. The effects of unemployment: A neglected problem in modern social researchs. En R. K. Merton y otros (Eds.) Qualitative and quantitative social research. New York: Free Press, 1979.

JAHODA, M. The impact of unemployment in the 1930s and the 1970s. Bulletin of the British Psychological Society. 1979, 32, 309-314.

JANOFF-Bulman, R. Charactereological versus behavioral self-blame: Inquiries into depression and rape. Journal of Personality and Social Psychology. 1979, 37, 1798-1809.

Kfilley, H. H. The processes of causal attribution. American Psychologist. 1973, 28, 107-128.

KuIPER, N. A, Depression and causal attributions for success and failure. Journal of Personality and Social Psychology, 1978, 36, 236-246.

MiLler, I. W. y NORMAN, W. H. Learned helplessness in humans: A review and attribution-theory model. Psychological Bulletin, 1979, 86, 93-118.

Nie, N. H., Hull, C. H. Jenkins, J. G., Strinbrenner, K. y Bent, D. H. SPSS: Statistical package for the social sitences. New York: McGraw-Hill, 1975. 


\section{Estudios}

Peterson, C., Schwartz, S. M. y Seligman, M. E. P. Self-blame and depressive symptoms. Journal of Personality and Social Psycbology, 41, 253-259.

Pitrman, N. L. y Pittman, T. S. Effects of amount of helplessness training and internal-external locus of control on mood and perfomance. Journal of Personality and Social Psycbology, 1979, 37, 39-47.

Rizley, R. Depression and distortion in the attribution of causality. Journal of Abnormal Psychology. 1978, 87, 32-48.

Roth, S. A revised model of learned helplessness in humans. Journal of Personality. 1980, 48, 103-133.

Seligman, M. E. P., Abramson, L. Y., Semmel, A y von Baeyer, C. Depressive attributional style. Journal of Abnormal Psychology, 1979, 88, 242-247.

VROOM, B. (Ed.) Work and motivation. New York: Wiley, 1964.

WeINER, B. (Ed.) Acbievement motivation and attribution tbeory. Morristown, N. J.: General Learning Press, 1974.

WeINER, B. Human motivation. New York: Holt, Rinehart \& Winston, 1980.

WeINER, B. An attributionally-based theory of motivation and emotion: Focus, range and issues. En N. T. Feather (Ed.) Expectations and actions: Expectancy-value models in psychology. Hillsdale, N. J., en prensa.

Weiner, B., Russell, D. y LERMAN, D. Affective consequences of causal ascriptions. En J. H. Harvey, W. Ickes y R. F. Kidd (Eds.) New directions in attribution research (vol. 2). Hillsdale, N.J.: Erlbaum, 1978.

WeINER, B., Russell, D. y LERMAN, D., The cognition-emotion process in achievement-related contexts. Journal of Personality and Social Psycbology, 1979, 37, 1211-1220.

WORTMAN, C. B. y BREHM, J. W. Responses to uncontrollable outcomes: An integration of reactance theory and the learned helplessness model. En L. Berkowitz (Ed.) Advances in experimental social psycho$\log y$ (vol. 8). Academic Press, 1975.

WORTMAN, C. B. y DiNTZER, J. Is an attributional analysis of the learned helplessness phenomenon viable? A critique of the Abramson-Seligman-Teasdale reformulation. Journal of Abnormal Psycbology, 1978, $87,75-90$. 